\title{
Influence of substrate biasing on the growth of c-axis oriented AIN thin films by $R F$ reactive sputtering in pure nitrogen
}

\author{
L. Monteagudo-Lerma ${ }^{*}$, , F.B. Naranjo ${ }^{1}$, M. González-Herráez ${ }^{1}$, S. Fernández ${ }^{2}$ \\ ${ }^{1}$ Departamento de Electrónica, Escuela Politécnica, Universidad de Alcalá, Campus Universitario, 28871 Alcalá de Henares, Spain \\ ${ }^{2}$ Departamento de Energías Renovables, Energía Solar Fotovoltaica, Centro de Investigaciones Energéticas, Medioambientales y Tec- \\ nológicas (CIEMAT), Avda. Complutense 22, 28040 Madrid, Spain
}

Received ZZZ, revised ZZZ, accepted ZZZ

Published online ZZZ (Dates will be provided by the publisher.)

Keywords (Nitrides, Semiconductor III-V materials, RF reactive sputtering, X-ray diffraction)

* Corresponding author: e-mail laura.monteagudo@depeca.uah.es, Phone: +34 918856908

\begin{abstract}
We report on the investigation of the influence of deposition conditions on structural, morphological and optical properties of AlN thin films deposited on sapphire $\left(\mathrm{Al}_{2} \mathrm{O}_{3}\right)$ substrates by radio-frequency $(\mathrm{RF})$ reactive sputtering. The deposition parameters studied are RF power, substrate temperature and substrate bias, while using pure nitrogen as reactive gas. The effect of such deposition parameters on AlN film properties are analyzed by different characterization methods as high resolution X-ray diffraction (HRXRD), field emission scanning electron microscopy (FESEM) and linear optical transmission. AIN
\end{abstract}

1 Introduction Aluminum nitride (AlN) has received considerable interest due to its important properties such as a wide direct band gap $(\sim 6.2 \mathrm{eV})$ [1], high thermal conductivity, high acoustic velocity and piezoelectricity. These properties make AlN material appropriated for a wide variety of applications in optoelectronics (UV spectral range), in surface acoustic wave devices (SAW) and in microelectromechanical systems (MEMS) [2]. In most of these applications, a highly quality AlN film with smooth surface is required.

Many techniques, such as metalorganic vapour phase epitaxy (MOVPE) and molecular beam epitaxy (MBE) are used to obtain AlN thin films on different substrates. The growth of AlN layers usually requires the use of high substrate temperature, which could deteriorate the substrate quality and thus the AlN properties during the growth process [3]. Hence, among the growth techniques generally used for AlN, RF reactive magnetron sputtering emerges as

\begin{abstract}
thin films with a full-width at half-maximum (FWHM) of the rocking curve obtained for the (0002) diffraction peak of $1.2^{\circ}$ are achieved under optimized conditions. The time resolved evolution of the self and externally-induced biasing of the substrate during deposition process is monitored and analyzed in terms of the rate of atomic species incorporation into the layer. The bias-induced change of the atomic incorporation leads to an enhancement in the structural quality of the layer and an increase of the deposition rate.
\end{abstract}

Copyright line will be provided by the publisher

a low-cost solution which allows deposition in both rigid and flexible substrates and in a wide range of temperatures.

In this work, we present the successful growth of AlN layers on sapphire by RF reactive sputtering under pure nitrogen atmosphere. Firstly, we discuss the effect of various deposition parameters, such as RF power and substrate temperature on crystalline structure, surface morphology and optical properties of AlN films. Besides, the effect of substrate biasing during deposition on the thickness, strain, grain size and apparent bandgap energy is analyzed.

2 Experimental The samples studied in this work consist of AlN layers deposited on $500 \mu \mathrm{m}$-thick c-axis oriented sapphire substrates using an RF sputtering system equipped with a 2" confocal magnetron cathode (AJA International, ATC ORION-3-HV). A high-purity Al disk (5N) was used as the $\mathrm{Al}$ target whereas the reactive gas was pure $\mathrm{N}_{2}(6 \mathrm{~N})$. The target-substrate distance was kept at 
$10.5 \mathrm{~cm}$ for all samples. A thermocouple placed in contact with the substrate holder allowed the monitorization of substrate temperature during deposition processes. Prior to growth, the substrates were cleaned in organic solvents and blown dry with nitrogen. Then, they were loaded in the sputtering system, which is pumped down to a base pressure of $\sim 10^{-7} \mathrm{mBar}$. Substrates were outgassed in the deposition chamber for $30 \mathrm{~min}$ at a substrate temperature $100{ }^{\circ} \mathrm{C}$ above the growth temperature.

The structural characterization of the deposited AlN films was performed by high resolution X-ray diffraction (HRXRD). A Bruker D8-4C Advance X-ray diffractometer was used to analyze both the crystal orientation and the structural quality of the layers by $2 \theta / \omega$ and rocking curve around (0002) diffraction peak measurements, respectively. The layer grain size $(G)$ is estimated by means of the Sherrer formula

$$
G=\frac{0.9 \cdot \lambda}{\Delta 2 \theta} \cdot \cos \theta
$$

in which $\Delta 2 \theta$ is the FWHM of the $\mathrm{AlN}(0002)$ diffraction peak in the $2 \theta / \omega$ measurement, $\lambda$ is the $\mathrm{Cu} \mathrm{K} \alpha_{1}$ wavelength and $\theta$ the diffraction angle corresponding to the (0002) orientation. XRD characterization was also used to determine the layer thickness by performing grazing incidence X-ray reflectivity (XRR) measurements.

The morphological analysis was carried out by FESEM technique using a FEI Nova Nanosem 230 microscope with VCD detector.

The linear optical properties were studied from transmission measurements in the $180-2500 \mathrm{~nm}$ spectral range, obtained under normal incidence and at room temperature using a UV/VIS/NIR Perkin-Elmer Lambda 1050 scanning spectrophotometer. The AlN bandgap energy was calculated by considering a sigmoidal fitting of the optical absorption obtained from transmission measurements.

3 Results and discussion The sputtering parameters studied in this work for the deposition of AlN layers are summarized in Table 1.

Table 1 AlN sputtering parameters

\begin{tabular}{ll}
\hline Parameter & Value \\
\hline Deposition pressure & $3.5 \mathrm{mT}$ Torr \\
Reactive gas, $\mathrm{N}_{2}$ & $100 \%$ \\
RF power & $100,125,150,175 \mathrm{~W}$ \\
Substrate temperature & $350,400,450,500^{\circ} \mathrm{C}$ \\
Substrate bias & Floating, $+5,-5,-25 \mathrm{~V}$ \\
Deposition time & $2 \mathrm{~h}$ \\
\hline
\end{tabular}

As it is shown in Table 1, this work is focused on the investigation of the effect of three deposition parameters, the RF power, the substrate temperature and the substrate bias on the AlN layer quality. For all the experiments the deposition working pressure was fixed to 3.5 mTorr, using pure nitrogen as reactive gas. Both conditions were taken for previous results obtained in the growth optimization process of InN layers in the same deposition system [4].

3.1. Effect of RF power The effect of RF power on the AlN quality was studied through a set of samples deposited at RF powers ranged from 100 to $175 \mathrm{~W}$ (see Table $1)$. For this set of samples the substrate temperature are kept fixed to $400{ }^{\circ} \mathrm{C}$, while the samples are not externally biased (floating substrate configuration).

Figure 1 shows a representative 20/ $\omega$ scan around the (0002) X-ray reflection peak of the AlN. It must be pointed out that all the samples deposited to investigate the effect of RF power present the reflection peak associated with (0002)-oriented AlN without parasitic crystallographic orientations.

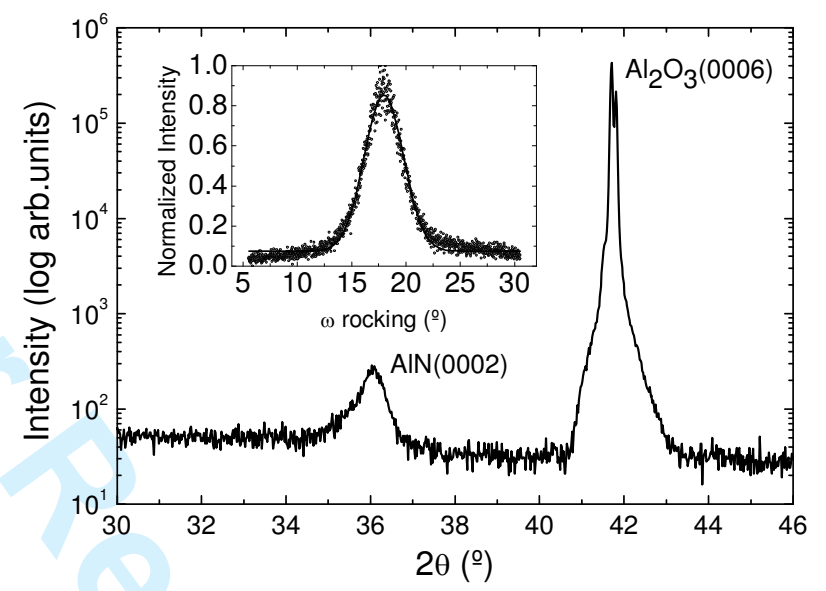

Figure 1 XRD $2 \theta / \omega$ scan of AlN films deposited on $\mathrm{Al}_{2} \mathrm{O}_{3}$ substrates at $100 \% \mathrm{~N}_{2}, 3.5 \mathrm{mTorr}, 100 \mathrm{~W}, 400{ }^{\circ} \mathrm{C}$ and floating substrate. Inset: XRD rocking curve of the $\mathrm{AlN}(0002)$ reflection peak fitted to a gaussian curve.

The estimated c-lattice parameter for AlN films under the previous deposition conditions is about $4.983 \AA$, which is close to the corresponding one to fully relaxed AlN films, of $4.982 \AA$ [5].

Figure 2 shows the $\omega$-scan XRD measurements of the set of samples deposited for this study. The FWHM of the rocking curve obtained for the $\mathrm{AlN}(0002)$ diffraction peak is decreased from $4.26^{\circ}$ to $2.18^{\circ}$ when increasing the deposition RF power from 100 to $150 \mathrm{~W}$. At the same time, an increase of the growth rate from 38 to $45 \mathrm{~nm} / \mathrm{h}$ is estimated using FESEM measurements when increasing the RF power from 100 to $150 \mathrm{~W}$, respectively. This improvement of crystal quality and growth rate with the RF power is in agreement with results presented by other authors [6][7]. It is worth saying that no deposition was detected for RF power below $100 \mathrm{~W}$. Further increase of this deposition parameter up to $175 \mathrm{~W}$ leads to a degradation of the layer crystalline quality. For this sample a FWHM of the (0002) 
diffraction peak in the $\omega$-scan of $3.8^{\circ}$ is obtained. This deterioration of crystal quality is attributed to surface damage generated by the high energy $\mathrm{Al}$ ions during deposition process.

At the same time, the estimated grain size of the studied AlN films follows a similar trend that the FWHM of the $\omega$-scan, with values of $14,19,26$ and $22 \mathrm{~nm}$ for RF powers of 100, 125, 150 and $175 \mathrm{~W}$, respectively. Taking into account the previous results, the RF power was fixed to $150 \mathrm{~W}$ for the following experiments.

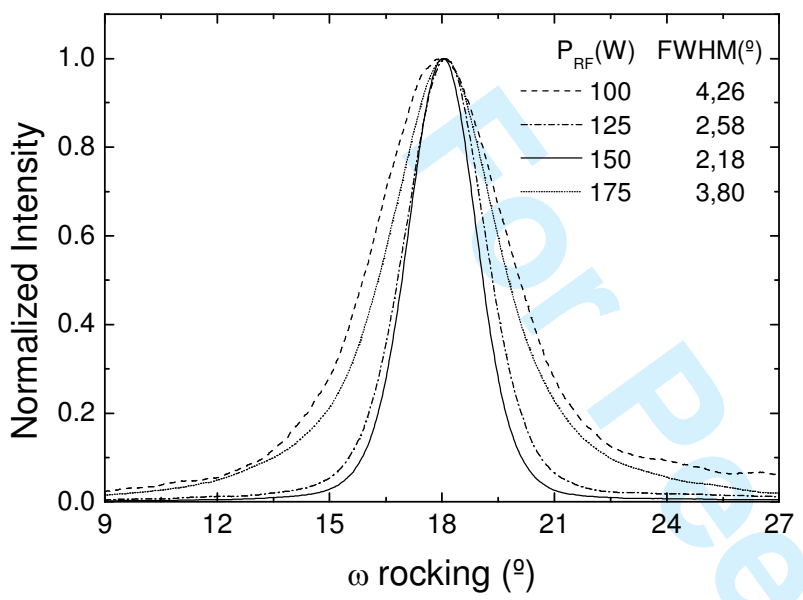

Figure 2 Normalized XRD $\omega$ scan of AlN films deposited on $\mathrm{Al}_{2} \mathrm{O}_{3}$ substrates at $100 \% \mathrm{~N}_{2}, 3.5 \mathrm{mTorr}, 400{ }^{\circ} \mathrm{C}$ and floating substrate as a function of the RF power.

3.2 Effect of substrate temperature For this study, the substrate temperature was changed in the range of $350{ }^{\circ} \mathrm{C}$ to $500{ }^{\circ} \mathrm{C}$. $2 \theta / \omega$ XRD patterns of the samples show that changes in substrate temperature do not have an influence on the preferred orientation of the layers. Regarding the structural quality, a slight decrease of the FWHM of the rocking curve is achieved from $2.18^{\circ}$ to $2.07^{\circ}$ at a temperature of $450{ }^{\circ} \mathrm{C}$. On the other hand, layers deposited at 350 and $500{ }^{\circ} \mathrm{C}$ show an increase of the FWHM to $8.77^{\circ}$ and $5.78^{\circ}$, respectively. The deterioration in the structural quality of the c-axis orientation growth for AlN films at higher temperature can be attributed to resputtering of atoms from the substrate [8]. Considering all these results, the optimized temperature to reach highquality c-axis oriented AlN thin films, under the previously fixed deposition conditions, is $450{ }^{\circ} \mathrm{C}$.

This optimized layer deposited at $100 \% \mathrm{~N}_{2}, 3.5$ mTorr, $150 \mathrm{~W}, 450{ }^{\circ} \mathrm{C}$ and floating substrate presents a c-axis lattice parameter of $4.973 \AA$. Thus, it shows a residual tensile strain $\left(\varepsilon_{\mathrm{zz}}\right)$ of $1.7 \cdot 10^{-3}$. The grain size is about $26 \mathrm{~nm}$ with a growth rate of $\sim 50 \mathrm{~nm} / \mathrm{h}$.

Figure 3 shows optical transmission measurements of the AlN layer deposited under optimized growth conditions. The absorption coefficient of the sample was derived from linear optical transmission (T) measurements using the re- lation $\alpha(\lambda) \propto-\ln (\mathrm{T})$, which neglects the losses introduced by interfaces reflection and optical scattering. The obtained absorption was modeled using a sigmoidal approximation that allows the bandgap energy estimation as the intersection of the linear fitting to $\alpha^{2}(\mathrm{E})$ with the $\mathrm{X}$-axis.

Figure 3 shows the above described fitting for the optimized sample, which leads to a bandgap estimation of 6.0 $\mathrm{eV}$, in good agreement with the obtained one in sputtered AlN samples [9].

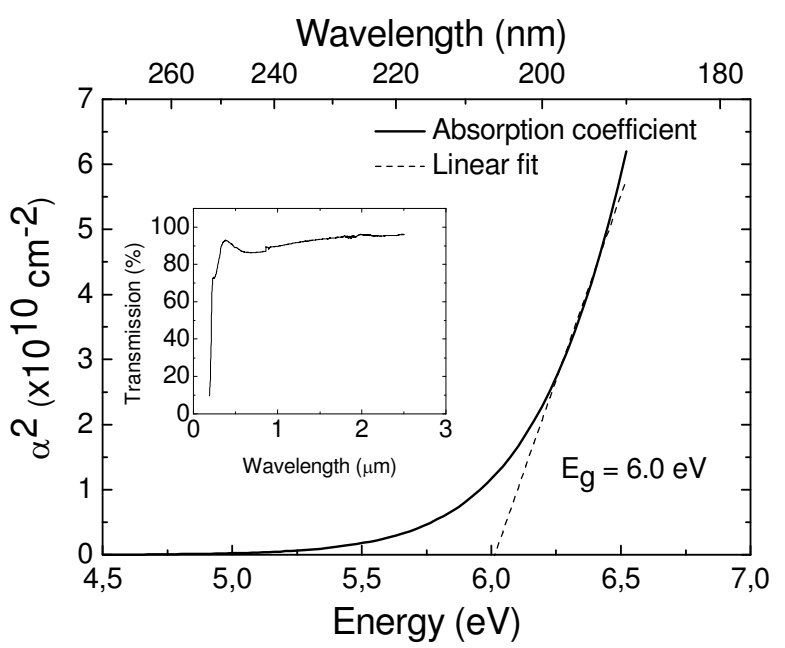

Figure 3 Linear fit of the experimental square absorption coefficient of AlN layer deposited onto c-axis sapphire substrates at $100 \% \mathrm{~N}_{2}, 3.5$ mTorr, $150 \mathrm{~W}, 450{ }^{\circ} \mathrm{C}$ and floating substrate. Inset: Optical transmission spectrum of the same sample in the 180$2500 \mathrm{~nm}$ spectral range.

3.3 Effect of substrate biasing Once AlN films are optimized in terms of RF power and substrate temperature, the third deposition parameter studied was the substrate biasing, which was changed between +5 to $-25 \mathrm{~V}$ (see Table 1).

For this experiment, a DC voltage source is connected to the substrate holder while the current intensity is measured as a function of deposition time. In the studied electrical configuration the deposition chamber is electrically grounded.

For all the biased samples, the measured timedependent evolution of the current intensity follows a similar behavior than one showed for a capacitor charging process. Namely, after an exponential growth, the current intensity saturates to a value which increases with the negative externally-induced bias (see Figure 4), changing from 0.4 to $2.5 \mathrm{~mA}$ for substrate bias of +5 to $-25 \mathrm{~V}$, respectively. This evolution and the dependence of the saturation intensity with the substrate bias is explained in terms of screening of the externally induced electric field in the sample from the impinging charged species present in the plasma during deposition. 


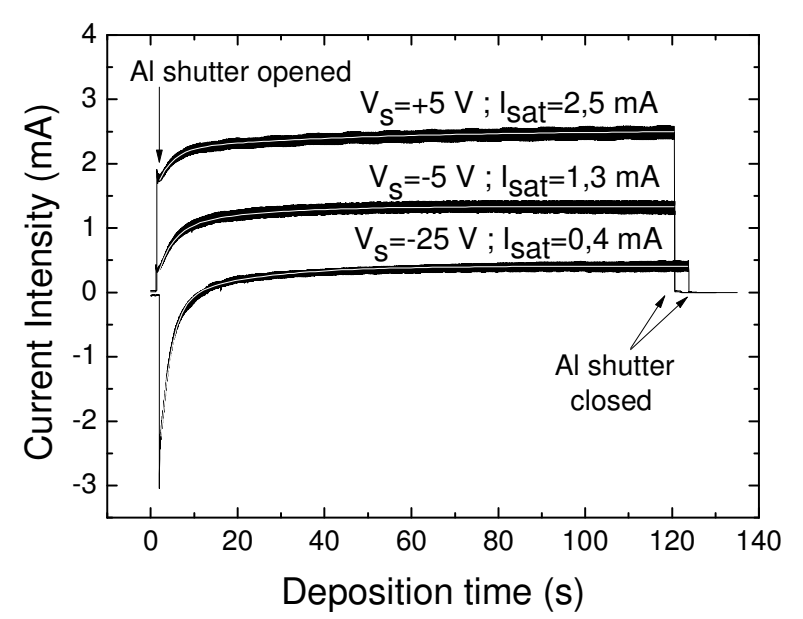

Figure 4 Real-time current intensity evolutions during depositions with external-induced bias as $+5 \mathrm{~V},-5 \mathrm{~V}$ and $-25 \mathrm{~V}$.

Figure 5 shows the $\omega$-scan XRD measurements of layers deposited under optimized deposition conditions and different substrate bias. A decrease of the FWHM values of the rocking curve is observed when increasing the negative substrate bias. This enhancement in the structural quality of the layers is related to the higher energy of the $\mathrm{Al}$ ions which reach the substrate under biasing conditions, enabling the migration of the adatoms at the growing surface. As it has been referred by other authors, the (0002) plane is favorable to grow when the atomic species have a high energy, as it involves the two types of bonds found in wurtzite structure [10].

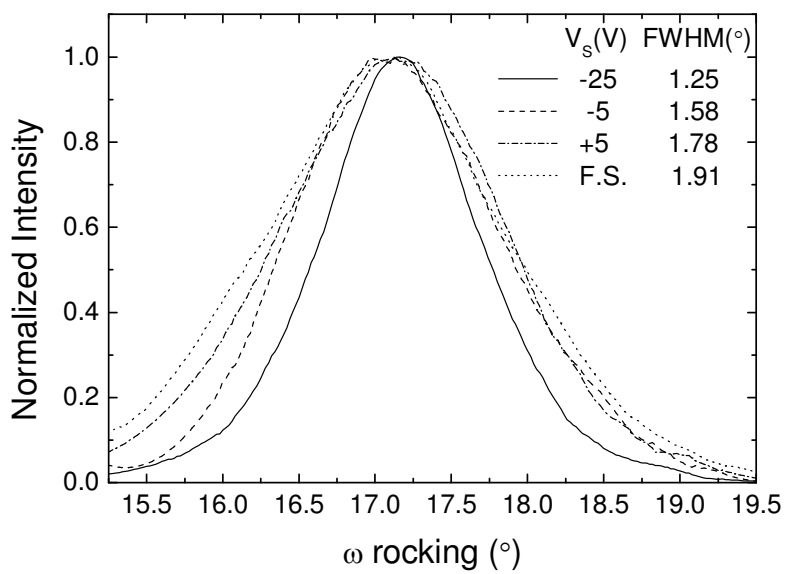

Figure $5 \omega$-scan XRD measurements of AlN films deposited on $\mathrm{Al}_{2} \mathrm{O}_{3}$ substrates at $100 \% \mathrm{~N}_{2}, 3.5 \mathrm{mTorr}, 150 \mathrm{~W}$ and $450{ }^{\circ} \mathrm{C}$ as function of the substrate bias applied, compared to the unbiased layer (in floating substrate configuration, F.S.).

Figure 6 shows the evolution of the grain size and the $\mathrm{c}$-axis lattice parameter depending on the applied substrate bias during AlN deposition. The highest grain size is ob- tained for substrate bias of $-25 \mathrm{~V}$, with a grain size of 52 $\mathrm{nm}$, which corresponds to a FWHM of the (0002) diffraction peaks obtained in $2 \theta / \omega$ scan of $1.2^{\circ}$, well below the obtained one of $1.9^{\circ}$ for samples grown without biasing the substrate.

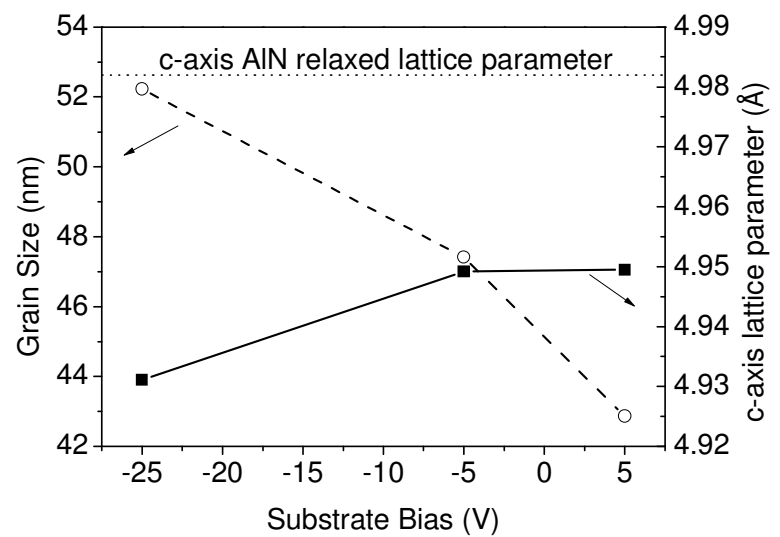

Figure 6 Dependence of grain size and c-axis lattice parameter on substrate bias for AlN layers deposited on sapphire at $100 \%$ $\mathrm{N}_{2}, 3.5$ mTorr, $150 \mathrm{~W}$ and $450{ }^{\circ} \mathrm{C}$.

The c-axis lattice parameter decreases with the negative substrate biasing from 4.949 to $4.931 \AA$ A (see Figure 6), which corresponds to tensile strain $\left(\varepsilon_{\mathrm{zz}}\right)$ states ranging from $6.6 \times 10^{-3}$ to $1.0 \times 10^{-2}$, respectively. This slightly increase in the residual strain in the layers is attributed to the increase of the grain size.

The layer thickness measured by XRR in grazing incidence condition is about $122 \mathrm{~nm}$, hence, the growth rate increases in almost $20 \%$ compared to the optimized unbiased layers, reaching a deposition rate of about $61 \mathrm{~nm} / \mathrm{h}$. Figure 7 show a FESEM image of AIN layer deposited under optimized deposition conditions with substrate bias, showing a compact morphology of the layer.

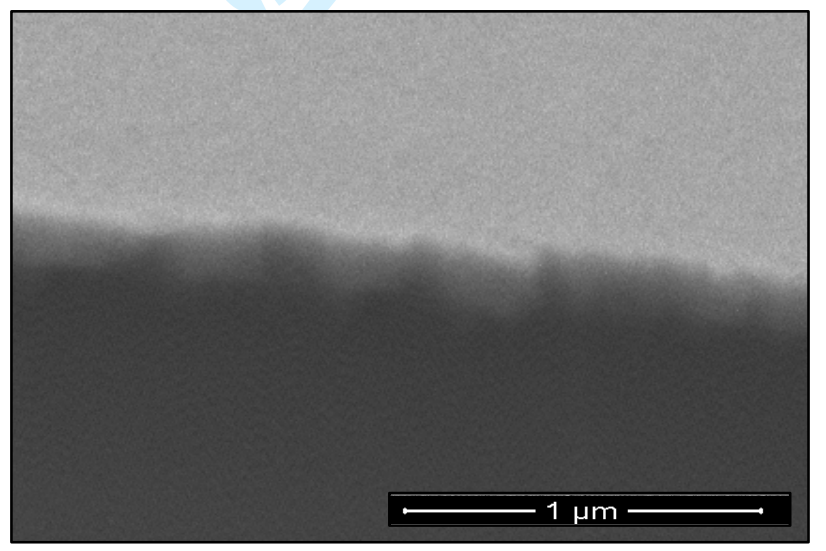

Figure 7 FESEM image of AlN layer deposited directly on caxis sapphire biased substrate at $-25 \mathrm{~V}$ and in a pure nitrogen atmosphere. The sputtering pressure, RF power and substrate temperature were $3.5 \mathrm{mTorr}, 150 \mathrm{~W}$ and $450^{\circ} \mathrm{C}$, respectively. 
Regarding the optical properties of the optimized AlN layers, a decrease of the estimated bandgap to $5.9 \mathrm{eV}$ is observed for biased samples, it being attributed to an enhanced incorporation of impurities.

3 Summary The influence of deposition conditions such as RF power, substrate temperature and substrate bias on morphological, structural and optical properties on AlN films deposited on (0006)-oriented $\mathrm{Al}_{2} \mathrm{O}_{3}$ in pure nitrogen by RF reactive sputtering were analyzed. AlN films with a (0002) preferred growth orientation were deposited in optimized sputtering conditions without substrate bias. For the analyzed conditions, the structural quality of the samples deteriorates for RF powers and deposition temperatures above $150 \mathrm{~W}$ and $450^{\circ} \mathrm{C}$, respectively.

AlN layers deposited biasing the substrate show an enhancement in crystalline quality on (0002) growth orientation. This result is attributed to an increase of the energy of the ions reaching the substrate due to the external biasing. Furthermore, for biased samples, the deposition rate increases while both grain size and residual tensile strain are enhanced.

Acknowledgements Partial financial support was provided by Spanish Gobermment Projects, TEC2009-14423-C02-02, and by Comunidad de Madrid Project P2009/ESP-1781.

\section{References}

[1] H. Cheng, Y. Sun, J.X. Zhang, Y.B. Zhang, S. Yuan and P. Hing, J. Crystal Growth 254, 46-54 (2003).

[2] C. Giordano, I. Ingrosso, M.T. Todaro, G. Maruccio, S. De Guido, R. Cingolani, A. Passaseo and M. De Vittorio, Microelec. Eng. 86, 1204-1207 (2009).

[3] H. Cheng, Y. Sun and P. Hing, Thin Solid Films 434 112120 (2003).

[4] S. Valdueza-Felip, F.B. Naranjo, M. González-Herráez, L. Lahourcade, E. Monroy and S. Fernández, Phys. Stat. Sol. A 208, No. 1, 65-69 (2011).

[5] M. E. Levinshtein, S. L. Rumyantsev, and M. S. Shur, Properties of Advanced Semiconductor Materials: GaN, AlN, InN, BN, SiC, SiGe (Wiley, New York, 2001).

[6] Z. Vashaei, T. Aikawa, M. Ohtsuka, H. Kobatake, H. Fukuyama, S. Ikeda and K. Takada, J. Crystal Growth 311, 459-462 (2009).

[7] Q.X. Guo, T. Tanaka, M. Nishio and H. Ogawa, Vacuum 80, 716-718 (2006).

[8] Q.X. Guo, K. Yahata, T. Tanaka, M. Nishio and H. Ogawa, J. Crystal Growth 257, 123-128 (2003).

[9] J.A. Guerra, L. Montañez, O. Erlenbach, G. Galvez, F. De Zela, A. Winnacker and R. Weingärtner, Journal of Physics: Conference Series 274, 012113 (2011).

[10] X. Xu, H. Wu, C. Zhang and Z. Jin, Thin Solid Film 388, 62-67 (2011). 